\title{
人工智能在电气自动化控制中的应用研究
}

\author{
胡要林 \\ 华润水泥投资有限公司，广东深圳 518000
}

[摘要]近年来, 我国社会综合国力在多方面利好因素的影响下, 得到了显著的提升, 从而为各个领域的发展壮大创造了良好 的基础，有效的推动了社会科学技术水平的不断提升，为产业革命的全面落实给予了良好的辅助，促进了生产效率的快速提 高, 在这种形势下大量的新兴科学技术被人们研发出来, 在实践运用过程中取得了良好的成绩, 其中人工智能是最具代表性 的一种当前最先进的科学技术, 在人类社会发展中起到了至关重要的影响作用, 尤其是在物联网和车载系统领域的实践运用 过程中起到了非常重要的影响。将人工智能技术运用到电器自动化控制领域之中, 在促进电器自动化行业的稳步发展能够起 到积极影响作用。 [关键词]人工智能; 电气自动化; 应用

DOI：10.33142/sca.v3i8.3131 中图分类号: TU885 文献标识码：A

\section{Application and Research of Artificial Intelligence in Electrical Automation Control}

HU Yaolin

China Resources Cement Investment Co., Ltd., Shenzhen, Guangdong, 518000, China

\begin{abstract}
In recent years, under the influence of many favorable factors, Chinese social comprehensive national strength has been significantly improved, which has created a good foundation for the development of various fields, effectively promoted the continuous improvement of social science and technology level, provided good assistance for the comprehensive implementation of the industrial revolution, and promoted the rapid improvement of production efficiency. A large number of emerging science and technology have been developed by people and have achieved good results in the process of practical application. Among them, artificial intelligence is the most representative of the most advanced science and technology, which has played a crucial role in the development of human society, especially in the field of Internet of things and vehicle system. The application of artificial intelligence technology in the field of electrical automation control can play a positive role in promoting the steady development of electrical automation industry.
\end{abstract}

Keywords: artificial intelligence; electrical automation; application

\section{引言}

人工智能技术是现如今最为先进的一种自动化技术, 并且被人们大范围的运用到了电气自动化控制系统之中, 取 得了良好的成效, 受到了人们的广泛青睐。在整个电器自动化控制系统之中, 将人工智能技术加以合理地运用, 对于 推动电气设备自动化性能的提升具有重要的影响作用, 并且也可以增强智能化控制的整体水平。人工智能技术可以促进 电器自动化控制工作效率的显著提升, 所以针对电器自动化人工智能技术进行综合分析研究, 是具有较强的现实意义的。

\section{1 现代人工智能技术基本概念}

现代人工智能技术其实质就是由专业人员运用计算机信息技术，完成具有一定规模性的操作系统的研发和运用， 保证某机械设备可以在维持物理运行的过程中表现出良好的人工性和智能性。人工智能技术最为突出的特征就是综合 性和复杂性, 其最为重要的作用就是增强机械设备的模仿人工操作的整体水平。近年来, 在科学技术水平快速提升的 带动下, 使得人工智能技术得以显著的发展进步, 从而其适用性也在不断的扩展, 这样就为其未来持续良好发展创造 了重要的基础。其次, 运用人工智能技术生产出来的遗传编程、智能机械设备等研究成果在众多领域中的实践运用取 得了良好的成效。当代人工智能技术蕴含了诸多最先进的科学技术, 所以在实施管理工作的时候, 务必要充分结合各 方面实际情况和需要推进各项工作, 这样才能将管理工作的作用切实的发挥出来。所以, 为了保证人工智能技术的良 好发展, 需要专业研究人员和使用人员综合各方面需要, 挑选适合的管理模式。因为现代人工智能技术的运用往往都 需要依赖函数近似器, 所以在人工智能设备实际运转过程中, 往往其他专业信息技术无法进行干涉 ${ }^{[1]}$ 。 


\section{2 我国人工智能的应用优点}

\section{1 具有较好的安全性}

人工智能技术的实践运用过程中是需要互联网以及先进的电气系统的辅助的, 从而能够有效的提升人工智能技术 实用性, 促使人工智能技术能够切实的起到增强电子自动化系统综合性能的作用。结合以往设备运行状况, 企业也能 够从不同的角度利用有效的方式方法来增强整个系统的运行稳定性。因为电气自动化技术要想保证良好的持续发展, 那么最为重要的就是需要确保各项基础参数的准确性, 这样就对计算机设备的运行准确性提出了更高的要求, 而将人 工智能技术加以实践运用能够切实的解决上述问题, 促进计算机设备运行效率和计算准确性的显著提升。在科学技术 快速发展的带动下, 使得人工智能技术整体水平得到了显著的提升, 从而也增强了人工智能技术的实用性, 当下我国 人工智能技术还处在起步阶段, 并且正在朝着模拟人工智能的方向迈进 ${ }^{[2]}$ 。

\section{2 人工智能的性能非常好}

就现如今实际情况来说, 我国人工智能已经得到了大范围的运用, 将人工智能技术运用到电气工程领域之中, 不 但可以促进系统运行的整体效率, 并且还能够保证系统综合性能的提升。在人工智能技术快速发展的影响下, 使得电 气工程系统也随之得到了良好的进步。为了能够不断提升人工智能技术水平, 那么还需要我们加大力度针对人工智能 技术加以深入的研究分析, 这就需要我们重视计算机和互联网技术的运用和研发, 从而为电气工程领域的未来健康发 展创作良好的基础。

\section{3 人工智能需要被正常使用}

就当下实际情况来说，我国在实施计算机技术和人工智能技术研究创新工作的目的就是为了增强技术的实用性、 高效性和可操作性, 从而保证实践生产工作能够实现更多的经济收益的目的。而要想达到上述目标, 那么还需要重视 人工智能技术的实践运用的稳定性, 之后保证人工智能技术能够高效稳定的完成既定的工作目标, 那么才能确保其作 用充分的发挥出来, 为使用者创作更多的经济和社会收益。电气自动化系统涉及到的层面较多, 所以具有十分突出的 复杂性和综合性, 整个系统中涉及到众多的线路, 如果在实际操作过程中出现任何的失误, 那么都会引发严重的不良 后果。如果电器自动化系统运行出现任何的故障问题, 那么必然需要大量人员和资源的损害进行修复, 从而会导致系 统运行成本的增加。而如果能够将人工智能技术加以实践运用, 那么就可以将工作人员从巨大的工作量中摆脱出来, 从而切实的控制整体成本, 这也是科学技术发展所带来的社会发展动力 ${ }^{[3]}$ 。总的来说, 将人工智能技术加以合理的实践 运用, 可以有效的控制成本和能耗, 所以电气工程领域当前大量的专业人士都加大了人工智能技术的研究力度, 希望 能够促进技术水平的快速提升。

\section{3 人工智能在电气自动化控制中的应用现状}

在产业革命的影响下, 为自动化领域相关企业发展壮大带来了良好的机遇, 并且也形成了诸多的挑战, 企业要想 保证自身能够稳定持续发展, 那么最为重要的就是需要充分结合当前社会发展形式, 合理地运用最先进的生产技术和 管理模式, 在增强企业自身生产综合性能和管理工作水平的基础上, 为社会现代化生产创造良好的基础。当下我国电 气自动化领域中人们对于人工智能技术在电器自动化控制系统中的实践运用过程中所具有的重要性有了全面的认识, 都在积极的将人工智能大范围的运用到电器自动化领域之中, 并在实践中不断总结和壮大, 希望能够对原有老旧模式 的生产系统加以改进。第一, 在电器自动化控制系统之中运用人工智能技术, 可以利用计算机设备的计算方法来完成 对图纸的切实设计, 这样才能够有效的提升系统的运行效率, 尽可能的提高产品研发的效率效果。第二, 还可以运用 人工智能技术来对各项信息数据加以统一收集, 对系统运行加以模拟, 从而为电气系统运行提供准确的信息和数据, 综合数据发展形式来编制效果图。第三, 电气设备系统还可以完成对各个系统设备的监控, 如果发生任何的系统故障, 都能够第一时间进行报警, 并且查找导致故障的根源, 利用有效的方式方法加以解决 ${ }^{[4]}$ 。

\section{4 人工智能技术在电气自动化中的应用创新}

\section{1 人工智能技术学习能力的应用}

为了尽可能的提升自动化系统的实际操作和管理效率, 提升控制工作的准确性, 那么最为重要的就是需要切实的 对最先进的科学技术加以实践运用, 诸如: 在实施智慧建筑项目施工工作的过程中, 以往建筑各类设备都会选择电气 自动化设备, 诸如声控灯, 这类照明设备能够在感知声音之后开启照明, 这属于最为基础的一种自动化控制模式, 而 将智能化控制系统加以运用, 声控灯可以结合周边的光照环境来进行自行照明亮度的调节。 


\section{2 人工智能技术在电气设备操作中的应用分析}

就以往实际情况来说, 人工操作十分的普遍, 人为因素是引发电气设备运行故障的主要因素, 往往会遇到因为外 界因素的影响而发生操作失误的情况, 这样就会影响到电气设备自身价值的发挥, 甚至会引发诸多的危险事故的发生。 而将人工智能技术切实的运用到电气设备之中, 针对各项参数进行适当的调节来确保电气设备可以按照既定的设计运 行, 这样就能够在保证系统高效运行的基础上, 尽可能的避免人为因素造成不良影响, 推动电气自动化系统良好稳定 运行 ${ }^{[5]}$ 。

\section{3 人工智能技术的智慧化应用}

与以往老旧的自动化技术相对比来说，人工智能技术在诸多领域都展现出了智慧化的特征，在自动化系统中所表 现的智慧能力集中在下面几个方面: 第一, 加大力度针对传感器技术进行研究和创新, 确保信息数据的良好准确性, 设计技术拥有一定的创新性。第二, 嘉庆大数据技术的合理运用, 针对各类新的模块进行创新和利用。第三, 在电器 自动化系统中将最先进的技术设备加以运用, 促进生产效率的不断提升。

\section{4 人工智能技术在诊断故障中的应用}

在电气设备运行过程中, 往往会受到外界多方面因素的影响, 极易发生运行故障的情况。在设备运行故障发生之 前, 往往会出现一些警示, 工作人员可以结合实际情况来对设备故障情况加以判断。第一，工作人员可以结合自身实 践经验对故障根源进行综合分析, 第二, 是工作人员对电气设备进行全面检验之后对故障加以判断。将人工智能技术 引用到电器自动化控制系统之中, 在设备正常运行之后, 可以借助智能技术来完成对各个模块的操作, 这样就可以更 加准确高效的对故障加以判断。

\section{5 电气自动化控制中存在的问题}

(1) 系统干扰问题。由于企业设备众多, 环境复杂, 特别是高低压变频器, 在各地都可以看到低压 $200 \sim 500 \mathrm{kw}$ 逆变器被广泛使用，并且近年来高压逆变器已被广泛使用。谐波干扰严重的时候，影响弱电信号。

(2) 操作环境和自动控制设备大多是电子产品, 需要更高的环境要求。特别是 PLC 或 DCS 需要更高的环境温度、 湿度和灰尘含量, 并且受夏季高温的影响, 易发生 CPU 停机。

\section{6 促进人工智能技术发展的对策}

我国专业人士应当积极的结合国外先进电气自动化技术成功经验, 组建高水平专业工作团队, 推动技术研发工作 的全面发展。

\section{7 结束语}

将人工智能技术合理的运用到电器自动化控制系统之中, 不但可以提升设备操作效果, 并且还能够保证在最短的 时间内将故障加以排除，为生产工作的有序开展创造良好的基础。

\section{[参考文献]}

[1]刘奇巍. 人工智能技术在电气自动化控制中的运用 [J].科技创新与应用,2020(30): 161-162.

[2]李华军. 基于电气自动化控制中的人工智能技术分析 [J].电子世界,2019(20)：133-134.

[3]李永男,高任,金松林.人工智能技术在电气自动化中的应用 [J].集成电路应用, 2019, 36 (11) : 74-75.

[4] 肖占胜. 人工智能技术在电气自动化控制中的应用思考 $[\mathrm{J}]$ 。电子技术与软件工程, 2018 (20) : 106-107.

[5]蔡敏. 人工智能在电气自动化控制中的应用探析 [J].内燃机与配件, 2018 (18) : 195-197.

作者简介: 胡要林 (1986.3-), 毕业于: 江汉大学, 所学专业: 自动化, 当前就职于: 华润水泥投资有限公司, 职务: 副主任工程师，职称级别：中级工程师职称。 